\title{
Low temperature dephasing saturation from elastic magnetic spin disorder and interactions
}

\author{
George Kastrinakis \\ Institute of Electronic Structure and Laser (IESL), Foundation for Research and Technology - Hellas (FORTH), P.O. Box \\ 1527, Iraklio, Crete 71110, Greece*
}

(April 26, 2005)

\begin{abstract}
We treat the question of the low temperature behavior of the dephasing rate of the electrons in the presence of elastic spin disorder scattering and interactions. In the frame of a self-consistent diagrammatic treatment, we obtain saturation of the dephasing rate in the limit of low temperature for magnetic scattering, in agreement with the non-interacting case. The magnitude of the dephasing rate is set by the strength of the magnetic scattering rate. We discuss the agreement of our results with relevant experiments.
\end{abstract}

An important quantity in disordered electronic systems is the dephasing rate $\tau_{\phi}^{-1}$. It provides a measure of the loss of coherence of the carriers, but in the two-particle channel - c.f. eq. (1) below. Decoherence arises from coulombic interactions, scattering by phonons, magnetic fluctuations etc. The saturation of the dephasing rate at low temperature $T$ seen in numerous experiments ${ }^{1-13}$ has attracted a vigorous interest, especially given the longstanding theoretical prediction for a vanishing $\tau_{\phi}^{-1}$ as the temperature $T \rightarrow 0 .{ }^{14-21}$.

Previous theoretical studies ${ }^{14-25}$ have focused on the calculation of $\tau_{\phi}^{-1}$ in the absence of spin-scattering disorder. The majority of these studies predict, correctly, a vanishing $\tau_{\phi}^{-1}(T \rightarrow 0)$. Here we determine and calculate the factors which contribute to dephasing in the presence of spin-scattering disorder. The saturation obtained allows for the consistent elucidation of this puzzle.

In the presence of spin-less disorder, the cooperon (particle-particle diffusion correlator - c.f. fig. 1) is given by

$$
C_{0}(q, \omega)=\frac{1}{2 \pi N_{F} \tau^{2}} \frac{1}{D q^{2}-i \omega+\tau_{\phi}^{-1}} .
$$

$D$ is the diffusion coefficient, $N_{F}$ is the density of states at the Fermi level and $\tau^{-1}$ the total impurity scattering rate. We work in the diffusive regime $\epsilon_{F} \tau>1(\hbar=1), \epsilon_{F}$ being the Fermi energy.

With spin-disorder present, the cooperon becomes spin-dependent. The relevant terms $C_{i}$ are shown in fig. 1 . We start by giving the explicit form of these $C_{0,1,2}^{o}$ without a dephasing rate. $C_{0}^{o}$ acquires a finite spin-dependent term in the denominator, which is crucial for the determination of the dephasing rate - c.f. below.

For the case , $\tau_{S}^{-1}>0, \tau_{s o}^{-1}=0$ - with $\tau_{S}^{-1}$ the magnetic impurity scattering rate and $\tau_{s o}^{-1}$ the spin-orbit impurity scattering rate - the cooperons are given by

$$
\begin{array}{r}
C_{0}^{o}(q, \omega)=\frac{1}{2 \pi N_{F} \tau^{2}} \frac{1}{D q^{2}-i \omega+2 /\left(3 \tau_{S}\right)}, \\
C_{1,2}^{o}(q, \omega)=b_{1,2}\left\{\frac{1}{D q^{2}-i \omega+2 /\left(3 \tau_{S}\right)}-\frac{1}{D q^{2}-i \omega+2 / \tau_{S}}\right\},
\end{array}
$$

with $b_{1}=\left(3 \tau_{S} /(2 \tau)-2\right) /(2 u), b_{2}=1 / u, u=4 \pi N_{F} \tau^{2}$. Based on eqs. (2), we expect a saturation of the dephasing rate. The simple diffusion pole is "cut-off" by the constant terms proportional to $\tau_{S}^{-1}$. On symmetry grounds, the spin-conserving Coulomb interaction cannot eliminate these terms.

We emphasize that the impurity scattering considered is elastic, bulk-type. Interfacial impurity scattering, though similar to bulk-type, is expected to differ in detail.

To calculate the dephasing rate, we write down and solve the appropriate coupled equations for all three renormalized cooperons $C_{i}(q, \omega), i=0,1,2$. We note that usually the terms containing the factors $d_{i}$ and $h_{i}$ below are completely omitted. The equations are shown schematically in fig. 2:

$$
\begin{array}{r}
C_{0}=C_{0}^{o}+C_{0}^{o} Y_{0} C_{0}, \\
C_{1}=C_{1}^{o}+C_{1}^{o} W_{a}+C_{2}^{o} W_{b}, \\
C_{2}=C_{2}^{o}+C_{1}^{o} W_{b}+C_{2}^{o} W_{a},
\end{array}
$$

with 


$$
W_{a}=Y_{n} C_{1}+Y_{r} C_{2}, W_{b}=Y_{n} C_{2}+Y_{r} C_{1}
$$

and

$$
Y_{0}=\left(1+h_{0}\right) \Sigma_{0}+d_{0} \Sigma_{1}, Y_{n}=\left(1+h_{1}\right) \Sigma_{1}+d_{1} \Sigma_{0}, Y_{r}=\left(1+h_{2}\right) \Sigma_{2}+d_{2} \Sigma_{0} .
$$

In fig. 3 we show explicitly the components of the self-energy terms $Y$ - c.f. the figure legend for further details. Here, $h_{0}=h_{1}=h_{2}=-2 /\left(\pi \epsilon_{F} \tau\right), d_{0}=d_{1}=\left\{1 /\left(\epsilon_{F} \tau\right)+4 \pi\right\}\left\{\tau_{\text {so }}^{-1}-\tau_{S}^{-1}\right\} /\left(2 \pi^{2} \epsilon_{F}\right)$ and $d_{2}=-2\left\{1+2 \pi \epsilon_{F} \tau\right\}\left\{\tau_{s o}^{-1}-\right.$ $\left.\tau_{S}^{-1}\right\} /\left(\pi \epsilon_{F}\right)$. The terms containing the factors $d_{i}$ - with a spin impurity line either looping around the cooperon or crossing it - provide the coupling between the spin-independent and the spin-dependent cooperons, as they produce spin flipping - c.f. the spin configuration of $C_{2}$ in fig. 1 and the figure legend. Also, note the minus relative sign between $\tau_{s o}^{-1}$ and $\tau_{S}^{-1}$ in $d_{i}$, coming from the respective spin flipping disorder vertices ${ }^{26}$. This has the peculiar effect that for $\tau_{s o}=\tau_{S}$ the spin disorder signature disappears in $\tau_{\phi o}$ - c.f. eq. (11) below.

In the spirit of ref. ${ }^{15}$ we obtain for the basic components $\Sigma_{i}$ of the self-energy

$$
\Sigma_{i} \equiv \Sigma_{i}(q=0, \omega=0) \simeq-c_{o} \sum_{q} \int_{-\infty}^{\infty} d \omega^{\prime} \frac{C_{i}\left(q, \omega^{\prime}+i 0\right) \operatorname{Im} V\left(q, \omega^{\prime}+i 0\right)}{\sinh \left(\omega^{\prime} / T\right)}
$$

where

$$
V(q, \omega)=\frac{v_{q}}{1+v_{q} \Pi(q, \omega)}, \Pi(q, \omega)=\frac{N_{F} D q^{2}}{D q^{2}-i \omega}
$$

Here $v_{q}$ is the bare Coulomb interaction and $c_{o}=8 N_{F}^{2} \tau^{4}$.

In the foregoing we make the approximation ${ }^{15}$

$$
\int_{-\infty}^{\infty} d \omega \frac{F(\omega)}{\sinh (\omega / T)} \simeq T \int_{-T}^{T} d \omega \frac{F(\omega)}{\omega}
$$

The bosonic modes with energy greater than $T$ manifestly do not contribute to the self-energy and the dephasing process, as also emphasized in refs. ${ }^{17,21}$.

In the limit $\epsilon_{F} \tau \gg 1$, we can decouple a $2 \times 2$ system of equations involving only $C_{1,2}$, to facilitate the solution of eqs. (3-5). Then eq. (3) yields directly for the dephasing rate $\tau_{\phi o}^{-1}$ - note the index $o$ - in the denominator of $C_{0}$

$$
\tau_{\phi o}^{-1}(T)=-\frac{\Sigma_{0}+d_{0} \Sigma_{1}}{2 \pi N_{F} \tau^{2}}
$$

This equation means that the dephasing in the spin-dependent cooperon channels also contributes to the dephasing rate in the spin-independent channel. We note that in the limit $\epsilon_{F} \tau \gg 1$ the factor $d_{0}$ in (11) is the only one remaining among the $d_{0,1,2}$ and $h_{0,1,2}$ appearing in eqs. (3)-(5). But as shown below, the factor $d_{0} \Sigma_{1}$ is too small in comparison to the factors $\tau_{S}^{-1}, \tau_{s o}^{-1}$ in the denominator of $C_{0}$.

We look in detail at the case of pure magnetic scattering, i.e. $\tau_{S}^{-1}>0$ and $\tau_{s o}^{-1}=0$. In Appendix B we discuss the case of pure spin-orbit scattering.

Diagonalizing the system formed by eqs. $(4,5)$ yields for the cooperons $C_{1,2}$

$$
C_{1,2}(q, \omega)=S_{1,2}\left[\frac{1}{D q^{2}-i \omega+R_{-}}-\frac{1}{D q^{2}-i \omega+R_{+}}\right]
$$

Here $M_{1}=\left[b_{1} m_{1} m_{2}+\left(b_{2}^{2}-b_{1}^{2}\right) m_{0} \Sigma_{1}\right] / m, M_{2}=\left[b_{2} m_{1} m_{2}-\left(b_{2}^{2}-b_{1}^{2}\right) m_{0} \Sigma_{2}\right] / m, R_{ \pm}=\left[m_{1} m_{2} \pm m_{0}\left(b_{2} \mp b_{1}\right)\left(\Sigma_{1} \mp\right.\right.$ $\left.\left.\Sigma_{2}\right)\right] / m, X=2\left(b_{2} \Sigma_{1}+b_{1} \Sigma_{2}\right), m_{1}=2 /\left(3 \tau_{S}\right), m_{2}=2 / \tau_{S}, m_{0}=m_{2}-m_{1}, m=m_{1}+m_{2}$. The derivation of eqs. (12) can be found in Appendix A. We note that it is important to keep the term $b_{j}\left(D q^{2}-i \omega\right)$ in $S_{j}$, to arrive at the correct solution. Further, eqs. (12) are valid in any dimension.

In $2-\mathrm{D}, v_{q}=2 \pi e^{2} / q$, and, following ${ }^{15,18}$, we take $V(q, \omega)=\frac{2 \pi e^{2}}{q} \frac{D q^{2}-i \omega}{D \kappa q-i \omega}$, with $\kappa=4 \pi N_{F} e^{2}$.

To calculate the self-energies, we first evaluate the integral in eq. (10)

$$
I_{j}(q)=\int_{-T}^{T} d \omega \frac{b_{j}\left(D q^{2}-i \omega\right)+M_{j}}{(D \kappa q)^{2}+\omega^{2}}\left\{\frac{1}{D q^{2}-i \omega+R_{-}}-\frac{1}{D q^{2}-i \omega+R_{+}}\right\} .
$$

Since we are interested in the low $T$ limit, we take 


$$
D q^{2}>T
$$

obtaining

$$
I_{j}(q) \simeq 2 T \frac{b_{j} D q^{2}+M_{j}}{(D \kappa q)^{2}}\left\{\frac{1}{D q^{2}+R_{-}}-\frac{1}{D q^{2}+R_{+}}\right\}
$$

Subsequently, we evaluate

$$
\int_{\sqrt{T / D}}^{\sqrt{z / D}} I_{j}(q)(\kappa-q) q d q
$$

with

$$
z=\max D q^{2}=\tau^{-1} .
$$

Finally we obtain the following equations for the self-energies

$$
\begin{array}{r}
\Sigma_{j}=\frac{T^{2} a_{2 D}}{X}\left\{\frac{M_{j}-b_{j} R_{+}}{R_{+}}\left[\kappa \ln \left(\frac{R_{+}+z}{R_{+}+T}\right)-2 \sqrt{\frac{R_{+}}{D}}\left(\arctan \sqrt{\frac{z}{R_{+}}}-\arctan \sqrt{\frac{T}{R_{+}}}\right)\right]\right. \\
\left.-\frac{M_{j}-b_{j} R_{-}}{R_{-}}\left[\kappa \ln \left(\frac{R_{-}+z}{R_{-}+T}\right)-2 \sqrt{\frac{R_{-}}{D}}\left(\arctan \sqrt{\frac{z}{R_{-}}}-\arctan \sqrt{\frac{T}{R_{-}}}\right)\right]+\kappa M_{j}\left(\frac{1}{R_{-}}-\frac{1}{R_{+}}\right) \ln \left(\frac{z}{T}\right)\right\},
\end{array}
$$

with $j=1,2$ and $a_{2 D}=2 c_{o} e^{2} /\left(D \kappa^{2}\right)$.

The solution is

$$
\Sigma_{j}=s_{j} T^{2}, s_{j}=b_{j} a_{2 D} \tau_{S}\left\{\sqrt{\frac{2}{D \tau_{S}}} \arctan \sqrt{2 z \tau_{S}}-\kappa \ln \left(1+2 z \tau_{S}\right)\right\},
$$

which is valid for

$$
\Sigma_{j} \ll 1 /\left(2 \tau_{S}\right)
$$

This condition accompanies the solutions for $\Sigma_{j}$ in 1-D and 3-D as well.

We consider the so-called weak localization contribution to the conductivity, given by the sum of ${ }^{26}$

$$
\delta \sigma_{o}=-\frac{e^{2} D u}{\pi} \sum_{q}\left\{C_{0}(q, 0)+C_{2}(q, 0)\right\},
$$

which in a magnetic field $H$ perpendicular to the 2-D system becomes

$$
\begin{gathered}
\delta \sigma_{o}=-\frac{e^{2} D u}{\pi} \frac{e H}{\pi} \sum_{n=0}^{N_{H}}\left\{\frac{1}{2 \pi N_{F} \tau^{2}} \frac{1}{4 D e H(n+1 / 2)+2 /\left(3 \tau_{S}\right)+\tau_{\phi o}^{-1}}\right. \\
\left.+\frac{4 b_{2} e D H(n+1 / 2)+M_{2}}{X}\left(\frac{1}{4 D e H(n+1 / 2)+R_{-}}-\frac{1}{4 D e H(n+1 / 2)+R_{+}}\right)\right\},
\end{gathered}
$$

with $N_{H}=1 /(4 \operatorname{De\tau } H)$.

If this formula were fit to the 2-D formula without magnetic (or spin-orbit) scattering

$$
\delta \sigma_{o}=-\frac{2 e^{3} D H}{\pi^{2}} \sum_{n=0}^{N_{H}} \frac{1}{4 D e H(n+1 / 2)+\tau_{\phi}^{-1}} .
$$

saturation of the dephasing is obtained due (mostly) to the factor $2 /\left(3 \tau_{S}\right)$ in the denominator of $C_{0}$. The factors $R_{ \pm}$in eq. (22) satisfy $R_{ \pm}=1 /\left(2 \tau_{S}\right)+O\left(T^{2}\right)$. As a result, the contribution of the $C_{2}$ term is small, because $R_{+}-R_{-}=O\left(T^{2}\right)$. The same applies to $1-\mathrm{D}$ and $3-\mathrm{D}$, with the power law being $T^{3 / 2}$ in $1-\mathrm{D}$, as shown below.

In 1-D, $v_{q}=2 e^{2} \ln \left(q_{m} / q\right), q_{m}$ being the inverse of the largest transverse dimension (width) of the system. Here, $\operatorname{Im} V(q, \omega)=-\frac{4 e^{4} N_{F} \omega D q^{2} \ln ^{2}\left(q_{m} / q\right)}{\omega^{2}+\left(D q^{2}\right)^{2}\left[1+2 e^{2} N_{F} \ln \left(q_{m} / q\right)\right]^{2}}$. 
To calculate the self-energies in eq. (8), we first evaluate the integrals

$$
\begin{aligned}
& L_{j}(\omega)=\int_{0}^{z} d x \frac{\sqrt{x}\left(b_{j}(x-i \omega)+M_{j}\right)}{B^{2} x^{2}+\omega^{2}}\left\{\frac{1}{x-i \omega+R_{-}}-\frac{1}{x-i \omega+R_{+}}\right\} \\
&=-2 \frac{\left(M_{j}-b_{j} R_{-}\right) \sqrt{R_{-}-i \omega} \arctan \sqrt{\frac{z}{R_{-}-i \omega}}}{\left\{B^{2}\left(R_{-}-i \omega\right)^{2}+\omega^{2}\right\}} \\
&+2 \frac{\left(M_{j}-b_{j} R_{+}\right) \sqrt{R_{+}-i \omega} \arctan \sqrt{\frac{z}{R_{+}-i \omega}}}{\left\{B^{2}\left(R_{+}-i \omega\right)^{2}+\omega^{2}\right\}} \\
&+\frac{\left\{i M_{j} B+b_{j} \omega(B-1)\right\} \arctan \sqrt{\frac{i B z}{\omega}}}{B^{3 / 2} \sqrt{-i \omega}}\left\{\frac{1}{i B R_{-}+\omega(B-1)}-\frac{1}{i B R_{+}+\omega(B-1)}\right\} \\
&+\frac{\left\{i M_{j} B+b_{j} \omega(B+1)\right\} \arctan \sqrt{\frac{-i B z}{\omega}}}{B^{3 / 2} \sqrt{i \omega}}\left\{\frac{1}{i B R_{-}+\omega(B+1)}-\frac{1}{i B R_{+}+\omega(B+1)}\right\} .
\end{aligned}
$$

Here $x=D q^{2}, z=\max x=\tau^{-1}$ as before, and we approximated the term $\ln \left(q_{m} / q\right)$ by its average, taking

$$
B=1+e^{2} N_{F}<\ln \left(q_{m}^{2} / q^{2}\right)>>1 .
$$

Then, we consider the low $T$ limit $z \gg T>|\omega|$, obtaining

$$
L_{j}(\omega)=\frac{M_{j} \pi}{\sqrt{2 \omega B^{3}}}\left\{\frac{1}{R_{-}}-\frac{1}{R_{+}}\right\}+\text {const. }
$$

and we evaluate

$$
\int_{-T}^{T} L_{j}(\omega) d \omega
$$

Thus we obtain the self-energy equations

$$
\Sigma_{j}=\frac{T^{3 / 2} a_{1 D} M_{j}}{X}\left\{\frac{1}{R_{-}}-\frac{1}{R_{+}}\right\},
$$

with $j=1,2$, and $a_{1 D}=\sqrt{2} c_{o}(B-1)^{2} /\left(N_{F} B^{3 / 2}\right)$. The solution is

$$
\Sigma_{j}=s_{j} T^{3 / 2}, s_{j}=\frac{\sqrt{2} b_{j} \tau_{S} c_{o}(B-1)^{2}}{N_{F} B^{3 / 2}} .
$$

In 3-D we take $V(q, \omega)=\frac{4 \pi e^{2}}{q^{2}} \frac{D q^{2}-i \omega}{P-i \omega}$, with $P=4 \pi e^{2} N_{F} D$. To calculate the self-energies of eq. (8), we first evaluate the integrals

$$
\begin{array}{r}
K_{j}(q)=\int_{-T}^{T} d \omega \frac{b_{j}\left(D q^{2}-i \omega\right)+M_{j}}{P^{2}+\omega^{2}}\left\{\frac{1}{D q^{2}-i \omega+R_{-}}-\frac{1}{D q^{2}-i \omega+R_{+}}\right\} \\
\simeq 2 T \frac{b_{j} D q^{2}+M_{j}}{P^{2}}\left\{\frac{1}{D q^{2}+R_{-}}-\frac{1}{D q^{2}+R_{+}}\right\} .
\end{array}
$$

Then, evaluating

$$
\int_{\sqrt{T / D}}^{\sqrt{z / D}} K_{j}(q)\left\{D q^{2}-P\right\} d q
$$

yields the self-energy equations

$$
\begin{array}{r}
\Sigma_{j}=\frac{T^{2} a_{3 D}}{X}\left\{\frac{\left(M_{j}-b_{j} R_{+}\right)\left(P+R_{+}\right)}{\sqrt{D R_{+}}} \arctan \sqrt{\frac{z}{R_{+}}}-\frac{\left(M_{j}-b_{j} R_{-}\right)\left(P+R_{-}\right)}{\sqrt{D R_{-}}} \arctan \sqrt{\frac{z}{R_{-}}}\right. \\
\left.+b_{j}\left(R_{+}-R_{-}\right) \sqrt{\frac{z}{D}}\right\} .
\end{array}
$$


Here $a_{3 D}=4 c_{o} e^{2} /\left(\pi P^{2}\right)$. We obtain the solution

$$
\Sigma_{j}=s_{j} T^{2}, s_{j}=\frac{b_{j} a_{3 D}}{2 \sqrt{D}}\left\{\sqrt{z}-\sqrt{2 \tau_{S}}\left(\frac{1}{2 \tau_{S}}+P\right) \arctan \sqrt{2 \tau_{S} z}\right\} .
$$

From the above we see that $\tau_{\phi o}^{-1}(T \rightarrow 0)$ is much smaller than both $\tau^{-1}$ and $\tau_{s p}^{-1}=\tau_{s o}^{-1}+\tau_{S}^{-1}$. Actually, $\tau_{s p}^{-1}$ dominates over $\tau_{\phi o}^{-1}(T \rightarrow 0)$ in the denominator of $C_{0}$, causing a 'saturation' of this contribution.

Besides the spin-scattering rate terms appearing in the denominator of $C_{0}$, the dephasing rate probed in experiments is also set by the terms $R_{ \pm}$- c.f. eq. (22), pure magnetic scattering case. As mentioned in Appendix $\mathrm{B}$, it is expected that in the presence of the Coulomb interaction the simple diffusion pole in $C_{2}$ - c.f. eq. (46) - survives intact, in the pure spin-orbit scattering case, thus yielding absence of dephasing saturation.

We note that the idea that magnetic scattering may cause saturation has been recently suggested in ref. ${ }^{13}$. The situation here is to be contrasted with the absence of spin disorder, where it has been shown that $\tau_{\phi}^{-1}(T \rightarrow 0) \rightarrow 0$, e.g. in 2-D $\tau_{\phi}^{-1}(T \rightarrow 0) \propto T \rightarrow 0^{14-21}$. Moreover, we should point out that other self-energy processes, which are first order in the interaction $V(q, \omega)$, e.g. with $V$ crossing diagonally the cooperon - c.f. e.g. ${ }^{17}$, do not modify qualitatively these results.

Now, the total correction to the conductivity can be written as

$$
\delta \sigma_{t o t}=\delta \sigma_{o}+\delta \sigma_{I}
$$

where the first term is the cooperon contribution of eq. (21) and the second term due to interactions, involving additional and more complicate terms. If $\delta \sigma_{I}(T \rightarrow 0)$ contains non-saturating terms, then the picture so far presented should change.

The majority of experiments show saturation of $\tau_{\phi}^{-1}$ in the low temperature limit. The samples in which saturation is observed probably contain magnetic impurities, even in minute quantities. Also, some of the samples, in which dephasing saturation is observed, are truly 2 -dimensional, such as wires in refs. ${ }^{2,3,6}$, quantum dots in refs. ${ }^{7,8}$ etc. We believe that the observed saturation can be understood in the frame of our results above, and should be due to magnetic scattering. Including the apparent lack of saturation in certain samples - e.g. ${ }^{6,13}$. In such cases it is difficult to say whether sufficiently low temperatures have been reached for saturation to be observable. The relevant $T$, below which saturation can be observed, is proportional to the strength of the magnetic scattering rate, and lack of saturation was observed in the cleaner samples - e.g. ${ }^{13}$.

In summary, we have demonstrated that, within the frame of our approach, dephasing saturation arises from magnetic disorder and interactions, with the role of the former being decisive. Already, without considering interactions ${ }^{26}$, the cooperon $C_{0}$ has a finite correction of the simple diffusion pole - c.f. eqs. (2) - which is equivalent to a 'saturating' dephasing rate, but not for the pure spin-orbit scattering case - c.f. eqs. (46). We treat the effects of interactions both on the spin-independent $C_{0}$ and on the spin-dependent $C_{1,2}$. Based on symmetry, a lack of saturation is expected for pure spin-orbit scattering. The magnitude of the 'dephasing rate' at $T \rightarrow 0$ is set by the magnetic scattering rate. It appears that our results are in agreement with relevant experiments.

The author has enjoyed useful discussions/correspondence with N. Birge, J. Bird, D.K. Ferry, D.E. Khmelnitskii, P. Kopietz, J.J. Lin, P. Mohanty, D. Natelson, P. Schwab and J. von Delft.

\section{APPENDIX A}

In the limit $\epsilon_{F} \tau \gg 1$ eqs. (4),(5) reduce to:

$$
\begin{aligned}
& C_{1}=C_{1}^{o}+C_{1}\left(\Sigma_{1} C_{1}^{o}+\Sigma_{2} C_{2}^{o}\right)+C_{2}\left(\Sigma_{1} C_{2}^{o}+\Sigma_{2} C_{1}^{o}\right), \\
& C_{2}=C_{2}^{o}+C_{1}\left(\Sigma_{2} C_{1}^{o}+\Sigma_{1} C_{2}^{o}\right)+C_{2}\left(\Sigma_{1} C_{1}^{o}+\Sigma_{2} C_{2}^{o}\right) .
\end{aligned}
$$

Then, setting $u_{1}=1-\left(\Sigma_{1} C_{1}^{o}+\Sigma_{2} C_{2}^{o}\right)$ and $u_{2}=\Sigma_{2} C_{1}^{o}+\Sigma_{1} C_{2}^{o}$,

$$
\text { Det }=u_{1}^{2}-u_{2}^{2}, D_{1}=C_{1}^{o} u_{1}+C_{2}^{o} u_{2}, D_{2}=C_{2}^{o} u_{1}+C_{1}^{o} u_{2},
$$

we have

$$
C_{j}=D_{j} / \text { Det }, j=1,2
$$

Taking 


$$
c=\frac{1}{B+m_{1}}-\frac{1}{B+m_{2}}, B=D q^{2}-i \omega, m_{1}=2 /\left(3 \tau_{S}\right), m_{2}=2 / \tau_{S},
$$

we obtain

$$
D_{j}=b_{j} c+(-1)^{j} c^{2}\left(b_{1}^{2}-b_{2}^{2}\right) \Sigma_{j}
$$

Then

$$
C_{j}=\frac{d_{j}}{N_{+} N_{-}}
$$

with $d_{j}=b_{j} m_{0}\left(B+m_{1}\right)\left(B+m_{2}\right)+(-1)^{j} m_{0}^{2}\left(b_{1}^{2}-b_{2}^{2}\right) \Sigma_{j}, N_{ \pm}=\left(B+m_{1}\right)\left(B+m_{2}\right)-m_{0}\left(b_{1} \Sigma_{1}+b_{2} \Sigma_{2}\right) \pm m_{0}\left(b_{2} \Sigma_{1}+b_{1} \Sigma_{2}\right)$ and $m_{0}=m_{2}-m_{1}$. We should emphasize that so far this algebra is exact.

In the following, we only keep terms of order $B$, obtaining :

$$
\begin{array}{r}
d_{j}=b_{j} m_{0}\left[m_{1} m_{2}+B\left(m_{1}+m_{2}\right)\right]+(-1)^{j} m_{0}^{2}\left(b_{1}^{2}-b_{2}^{2}\right) \Sigma_{j}, \\
N_{+}=m_{1} m_{2}+B\left(m_{1}+m_{2}\right)+m_{0}\left(b_{1}-b_{2}\right)\left(\Sigma_{2}-\Sigma_{1}\right), \\
N_{-}=m_{1} m_{2}+B\left(m_{1}+m_{2}\right)-m_{0}\left(b_{1}+b_{2}\right)\left(\Sigma_{2}+\Sigma_{1}\right) .
\end{array}
$$

Making use of

$$
\frac{1}{N_{+} N_{-}}=\frac{1}{m_{0} X}\left(\frac{1}{N_{-}}-\frac{1}{N_{+}}\right)
$$

we obtain directly eqs. (12).

The derivation above is equally straightforward for the case of spin-obit disorder.

\section{APPENDIX B}

In this Appendix we discuss the case of finite spin-orbit impurity scattering, i.e. $\tau_{s o}^{-1}>0, \tau_{S}^{-1}=0$. The cooperons are given by $^{26,27}$

$$
\begin{array}{r}
C_{0}^{o}(q, \omega)=\frac{1}{2 \pi N_{F} \tau^{2}} \frac{1}{D q^{2}-i \omega+4 /\left(3 \tau_{s o}\right)}, \\
C_{1,2}^{o}(q, \omega)=b_{1,2}^{\prime}\left\{\frac{1}{D q^{2}-i \omega}-\frac{1}{D q^{2}-i \omega+4 /\left(3 \tau_{s o}\right)}\right\}
\end{array}
$$

with $b_{1}^{\prime}=\left(3 \tau_{s o} / \tau-2\right) /(2 u), b_{2}^{\prime}=-1 / u, u=4 \pi N_{F} \tau^{2}$.

Diagonalizing the system formed by eqs. $(4,5)$ yields for the cooperons $C_{1,2}$

$$
C_{1,2}(q, \omega)=S_{1,2}^{\prime}\left[\frac{1}{D q^{2}-i \omega+r_{-}}-\frac{1}{D q^{2}-i \omega+r_{+}}\right] .
$$

Here $r_{+}=\left(b_{1}^{\prime}-b_{2}^{\prime}\right)\left(\Sigma_{2}-\Sigma_{1}\right), r_{-}=-\left(b_{1}^{\prime}+b_{2}^{\prime}\right)\left(\Sigma_{2}+\Sigma_{1}\right), S_{j}^{\prime}=\left\{b_{j}^{\prime}\left(D q^{2}-i \omega\right)+M_{j}^{\prime}\right\} / X^{\prime}, X^{\prime}=2\left(b_{1}^{\prime} \Sigma_{2}+b_{2}^{\prime} \Sigma_{1}\right)$, $M_{1}^{\prime}=\left(b_{2}^{\prime 2}-b_{1}^{\prime 2}\right) \Sigma_{1}, M_{2}^{\prime}=-\left(b_{2}^{\prime 2}-b_{1}^{\prime 2}\right) \Sigma_{2}$. The derivation of eqs. (47) is the same as for the magnetic impurity case given in Appendix A.

The diffusion pole in eqs. (46) survives, by symmetry, in the presence of the spin-conserving Coulomb interaction, which implies $r_{+}=0$ or $r_{-}=0$ in eqs. (47).

The 2-D weak localization correction to the conductivity is

$$
\begin{gathered}
\delta \sigma_{o}=-\frac{e^{2} D u}{\pi} \frac{e H}{\pi} \sum_{n=0}^{N_{H}}\left\{\frac{1}{2 \pi N_{F} \tau^{2}} \frac{1}{4 D e H(n+1 / 2)+4 /\left(3 \tau_{s o}\right)+\tau_{\phi o}^{-1}}\right. \\
\left.+\frac{4 b_{2}^{\prime} e D H(n+1 / 2)+M_{2}^{\prime}}{X}\left(\frac{1}{4 D e H(n+1 / 2)+r_{-}}-\frac{1}{4 D e H(n+1 / 2)+r_{+}}\right)\right\}
\end{gathered}
$$

with $N_{H}=1 /(4 \operatorname{De\tau } H)$. 
Fitting this expression to eq. (23) with either $r_{+}=0$ or $r_{-}=0$ yields absence of dephasing saturation. This is the case for all dimensionalities for pure spin-orbit scattering. As mentioned in the text, this is not the case for finite magnetic scattering.

* E-mail address: kast@iesl.forth.gr

${ }^{1}$ J.J. Lin and J. Bird, J. Phys.: Condens. Matter 14, R501 (2002).

${ }^{2}$ P. Mohanty, E.M.Q. Jariwala and R.A. Webb, Phys. Rev Lett. 78, 3366 (1997).

${ }^{3}$ P. Mohanty, Physica B 280, 446 (2000).

${ }^{4}$ J. Bird, A.D.C. Grassie, M. Lakrimi, K.M. Hutchings, J.J. Harris and C.T. Foxon, J. Phys.: Condens. Matter 2, 7847 (1990).

${ }^{5}$ M. Noguchi, T. Ikoma, T. Odagiri, H. Sakakibara and S.N. Wang, J. Appl. Phys. 80, 5138 (1996).

${ }^{6}$ D. Natelson, R.L. Willett, K.W. West, and L.N. Pfeiffer, Phys. Rev. Lett. 86, 1821 (2001).

${ }^{7}$ A.G. Huibers, J.A. Folk, S.R. Patel, C.M. Marcus, C.I. Duruõz and J.S. Harris, Phys. Rev. Lett. 83, 5090 (1999).

${ }^{8}$ D.P. Pivin, A. Andresen, J.P. Bird and D.K. Ferry, Phys. Rev. Lett. 82, 4687 (1999).

${ }^{9}$ A.B. Gougam, F. Pierre, H. Pothier, D. Esteve and N.O. Birge, J. Low Temp. Phys. 118, 447 (2000).

${ }^{10}$ J.J. Lin and K.Y. Kao, J. Phys.: Condens. Matter 13, L119 (2001).

${ }^{11}$ J.J. Lin, T.J. Li and Y.L. Zhong, J. Phys. Soc. Jpn. 72, Suppl. A, 7 (2003); and therein.

${ }^{12}$ F. Pierre and N.O. Birge, Phys. Rev Lett. 89, 206804 (2002).

${ }^{13}$ F. Pierre, A.B. Gougam, A. Anthore, H. Pothier, D. Esteve, and N.O. Birge, Phys. Rev. B 68, 085413 (2003).

${ }^{14}$ B.L. Altshuler, A.G. Aronov and D.E. Khmelnitskii, J. Phys. C 15, 7367 (1982).

${ }^{15}$ H. Fukuyama and E. Abrahams, Phys. Rev. B 27, 5976 (1983).

${ }^{16}$ I.L. Aleiner, B.L. Altshuler and M.E. Gershenzon, Waves Random Media 9, 201 (1999).

${ }^{17}$ I.L. Aleiner, B.L. Altshuler and M.G. Vavilov, J. Low Temp. Phys. 126, 1377 (2002); cond-mat/0110545.

${ }^{18}$ R. Raimondi, P. Schwab and C. Castellani, Phys. Rev. B 60, 5818 (1999).

19 A. Völker and P. Kopietz, Phys. Rev. B 61, 13508 (2000).

${ }^{20}$ A. Völker and P. Kopietz, Phys. Rev. B 65, 045112 (2002).

${ }^{21}$ J. von Delft, J. Phys. Soc. Jpn. 72, Suppl. A, 24 (2003); cond-mat/0210644.

${ }^{22}$ D.S. Golubev and A.D. Zaikin, Phys. Rev. B 59, 9195 (1999).

${ }^{23}$ D.S. Golubev, A.D. Zaikin and G. Schön, J. Low Temp. Phys. 126, 1355 (2002); cond-mat/0110495.

${ }^{24}$ Y. Imry, H. Fukuyama and P. Schwab, Europhys. Lett. 47, 608 (1999).

${ }^{25}$ A. Zawadowski, J. von Delft and D.C. Ralph, Phys. Rev. Lett. 83, 2632 (1999).

${ }^{26}$ G. Bergmann, Phys. Rep. 107, 1 (1984).

${ }^{27}$ The prefactors $b_{1,2}$ and $b_{1,2}^{\prime}$ in eqs. (46) and (2) are correct herein. In ref. ${ }^{26}$ they contain a superfluous factor. 

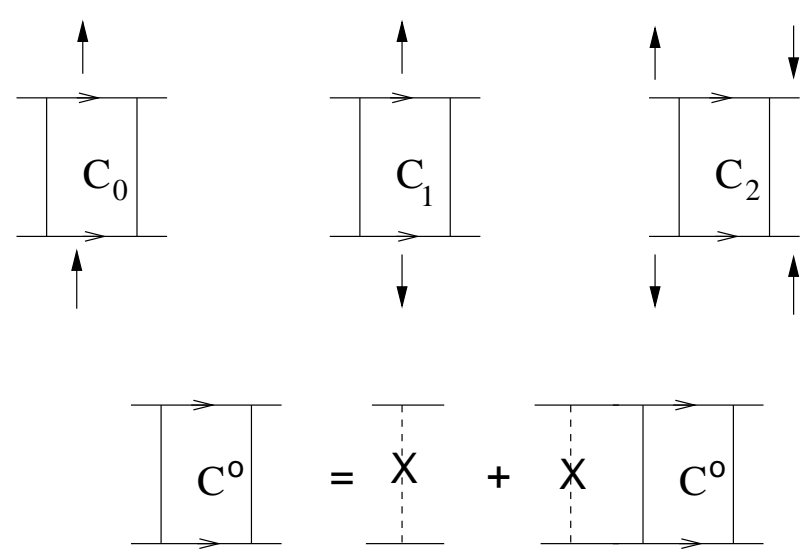

Fig. 1

The 3 cooperons $C_{0}, C_{1}, C_{2}$. Note the spin indices. The cooperons $C_{i}^{o}$ do not contain a dephasing rate. The dashed line with the cross stands for impurity (disorder) scattering. The bare disorder vertices flipping spin, corresponding to the spin configuration of $C_{2}$, yield a coupling of all three cooperons.

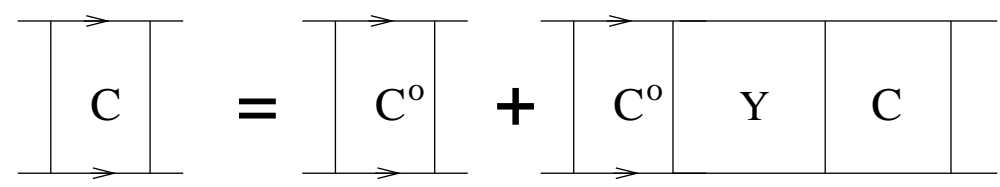

Fig. 2

Schematic form of the equations (3-5) involving the cooperons and the self-energies $Y$.

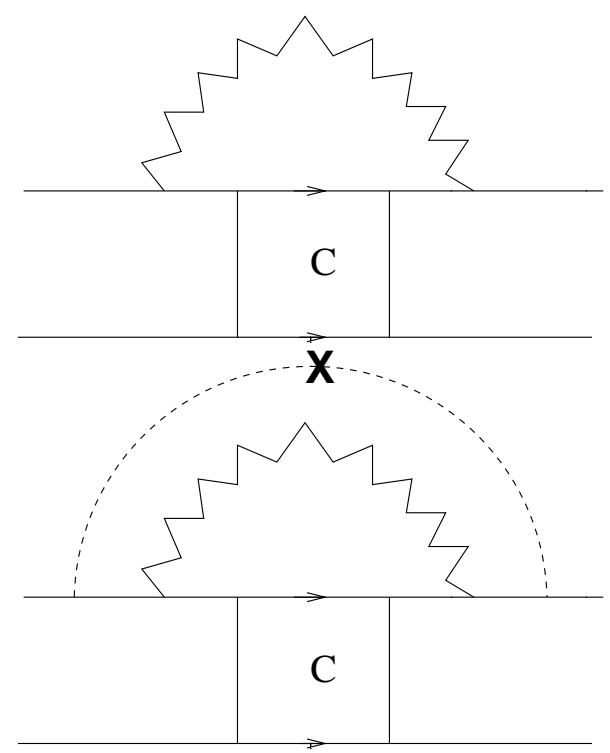



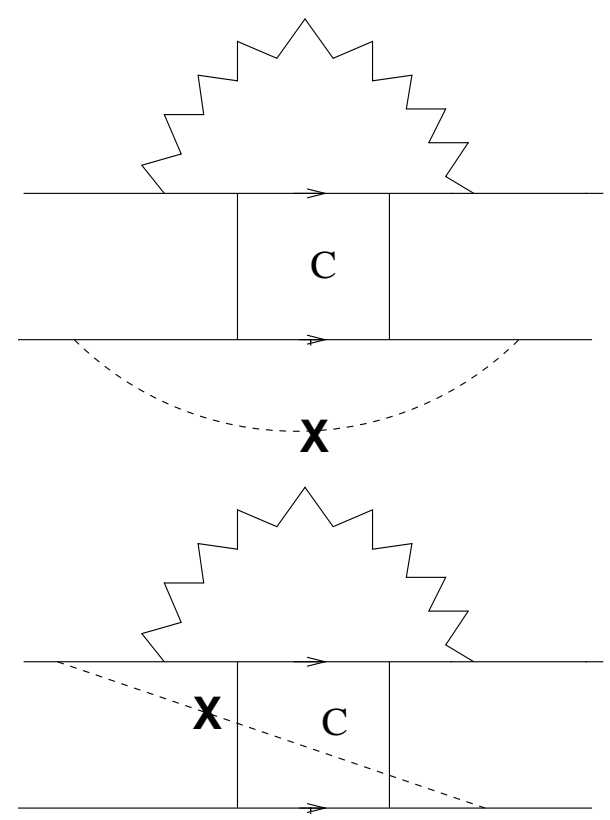

Fig. 3

The various components of the self-energy $Y$. The wiggly line represents the screened Coulomb interaction of eq. (9). The diagrams with the extra spin-disorder impurity line are responsible for the contribution $d_{0} \Sigma_{1}$ in $\tau_{\phi o}^{-1}$ (c.f. eq. (11)). We consider all possible variations of the diagrams shown here. That is, including terms in which the positions of the interaction and impurity vertices are interchanged along the particle lines. E.g. in the second diagram above, suppose we label the Coulomb and disorder vertices along the upper electron line by the numbers $(1,2,3,4)$. For this diagram we also consider the permutations $(2,1,3,4),(1,2,4,3)$ and $(2,1,4,3)$. 